\title{
Low-momentum Pion Enhancement Induced by Chiral Symmetry Restoration
}

\author{
Pengfei Zhuang \\ Physics Department, Tsinghua University, Beijing 100084, China
}

The thermal and nonthermal pion production by sigma decay and its relation with chiral symmetry restoration in a hot and dense matter are investigated. The nonthermal decay into pions of sigma mesons which are popularly produced in chiral symmetric phase leads to a low-momentum pion enhancement as a possible signature of chiral phase transition at finite temperature and density.

Keywords : chiral symmetry, pion spectra PACS : 11.30.Rd, 14.40. - n

As is well known, the ultimate goal of relativistic heavy ion collisions [1] is to study the deconfinement process in moving from a hadron gas to a quark-gluon plasma and the chiral transition from the chiral symmetry breaking phase to the phase in which it is restored [2]. In recent years some signatures of the chiral transition have been proposed, such as the excess of pions due to a rapid thermal and chemical equilibration in the symmetric phase [3], the excess of low energy photon pairs by pion annihilation in hot and dense medium [4], the dilepton enhancement from leptonic decay of sigma [5], and the enhancement of the continuum threshold in the scalar channel [6]. Most of the signatures are associated with the changes of sigma properties at finite temperatures and densities. While in the vacuum there is no obvious reason for the presence of sigma mesons in the study of chiral properties, there is a definite need [7] for sigma at finite temperatures and densities in the investigation of chiral symmetry restoration. With increasing temperature and density sigma changes its character from a resonance with large mass to a bound state with small mass. This medium dependence of sigma mass makes important sense not only in thermodynamics [8], but also in particle yields. Around the critical point of the chiral transition sigma is one of the most numerous species, since it is nearly massless. If the transition happens at the freeze-out these light sigmas will lead to large eventby-event fluctuations [9] of final state pions due to the $\sigma$-exchange in the pion thermodynamic potential [10].

A direct signature of these light sigmas in hot and dense matter is a nonthermal excess [10] of pions due to the decay process $\sigma \rightarrow 2 \pi$. With the expansion of the hadronic system produced in relativistic heavy ion collisions, the in-medium sigma mass rises towards its vacuum value and eventually exceeds the $\pi \pi$ threshold. As the $\sigma \pi \pi$ coupling is large, the decay proceeds rapidly. When this process occurs before freeze-out, the produced pions will be thermalized in the heat bath. However, when the decay happens after freeze-out, the generated pions do not have a chance to thermalize. Thus, it is expected $[10,11]$ that the resulting pion spectrum will have a nonthermal enhancement at low momentum.

The dependence of non-strangle hadron masses on temperature $T$ and baryon density $n$ can be well parameterized as $[12]$

$$
\frac{m(T, n)}{m(0,0)}=\left(1-\left(\frac{T}{T_{c}}\right)^{1 / 3}\right)\left(1-0.2 \frac{n}{n_{0}}\right),
$$

where $T_{c}$ is the critical temperature of chiral phase transition, and $n_{0}$ the baryon density of normal nuclear matter. From this parameterization, the temperature and density effects are quite different: The temperature dependence is very weak at low temperatures $\left(T / T_{c}<1 / 2\right)$, while the hadron masses decrease linearly in density in the whole density region. This difference between temperature and density effects is reflected in the pion spectra in high energy nuclear collisions. For ultra-relativistic heavy ion collisions where the central region is almost baryon free and the temperature effect is dominant, the sigma and pion masses are nearly constants when the temperature of the system is much below the critical one. Therefore, the pions produced from nonthermal sigma decay after freeze-out will have almost the same momentum $p_{0}=\sqrt{\frac{m_{\sigma}^{2}(0,0)}{4}-m_{\pi}^{2}(0,0)}$ in the vacuum. Aside from an overall enhancement of pions due to the thermal sigma decay, there will be also a peak in the pion spectra due to the nonthermal sigma decay. This peak will disappear partly when the density effect can not be neglected, since in this case the sigma mass varies significantly even after the freeze-out. Therefore, the sharp peak in the spectrum will be replaced by a smooth low-momentum pion enhancement.

We now illustrate the above statement by using a simple statistic model. The measured final-state pions include the direct pions emitted from the fireball and the pions from sigma decay,

$$
N=N_{d i r}+N_{d e c} .
$$

$N_{d i r}$ is determined at freeze-out, and $N_{d e c}$ is twice the number of sigmas at chiral phase transition where sigma has minimum mass and is then most numerous,

$$
\begin{aligned}
& N_{d i r}=V_{f} n_{\pi}\left(T_{f}\right), \\
& N_{d e c}=2 N_{\sigma}\left(T_{c}\right)=2 V_{c} n_{\sigma}\left(T_{c}\right),
\end{aligned}
$$

where $T_{f}$ and $V_{f}$ are the temperature and volume of the system at freeze-out, $T_{c}$ and $V_{c}$ the temperature and volume at chiral phase transition, $n_{\pi}\left(T_{f}\right)$ and $n_{\sigma}\left(T_{c}\right)$ the pion and sigma number densities at $T_{f}$ and $T_{c}$, 


$$
\begin{aligned}
& n_{\pi}\left(T_{f}\right)=\int \frac{d^{3} \mathbf{p}}{(2 \pi)^{3}} \frac{3}{e^{\epsilon_{\pi} / T_{f}}-1}, \\
& n_{\sigma}\left(T_{c}\right)=\int \frac{d^{3} \mathbf{p}}{(2 \pi)^{3}} \frac{1}{e^{\epsilon_{\sigma} / T_{c}}-1},
\end{aligned}
$$

with the pion and sigma energies $\epsilon_{\pi}\left(T_{f}\right)=\sqrt{m_{\pi}^{2}\left(T_{f}\right)+p^{2}}$ and $\epsilon_{\sigma}\left(T_{c}\right)=\sqrt{m_{\sigma}^{2}\left(T_{c}\right)+p^{2}}$. In central region of relativistic heavy-ion collisions where there is almost no net baryon and one considers the temperature effect only, the Bjorken's scaling hydrodynamics [13] is often used to describe the space-time evolution of the produced hot system. In this scenario the temperature $T_{f}$, volume $V_{f}$ and proper time $\tau_{f}$ at freeze-out and the corresponding quantities $T_{c}, V_{c}$ and $\tau_{c}$ at chiral phase transition have simple relations

$$
\begin{aligned}
& T_{f}=T_{c}\left(\frac{\tau_{c}}{\tau_{f}}\right)^{\frac{1}{3}}, \\
& V_{f}=V_{c} \frac{\tau_{f}}{\tau_{c}} .
\end{aligned}
$$

For central $A-A$ collisions with impact parameter $b=0$, the number of participant nucleons can be taken as $\left\langle N_{\text {part }}\right\rangle=2 A$ and the volume at freeze-out can be assumed to be proportional to $A, V_{f}=A v_{f}$. The pseudorapidity density of charged pions per participant pair in the mid-rapidity range $|\eta|<1$ is then written as,

$$
\begin{aligned}
& \left.\frac{d N}{d \eta}\right|_{\Delta \eta} / 0.5\left\langle N_{\text {part }}\right\rangle=\frac{1}{2} \frac{2}{3} v_{f} \times \\
& \left.\quad\left(1+2\left(\frac{T_{f}}{T_{c}}\right)^{3} \frac{n_{\sigma}\left(T_{c}\right)}{n_{\pi}\left(T_{f}\right)}\right) n_{\pi}\left(T_{f}\right)\right|_{\Delta \theta},
\end{aligned}
$$

where $\left.n_{\pi}\left(T_{f}\right)\right|_{\Delta \theta}$ is the pion number density in the angle interval $2 \arctan e^{-1}<\theta<2 \arctan e$,

$$
\left.n_{\pi}\left(T_{f}\right)\right|_{\Delta \theta}=\int_{\Delta \theta} \frac{d^{3} \mathbf{p}}{(2 \pi)^{3}} \frac{3}{e^{\epsilon_{\pi} / T_{f}}-1},
$$

and the factor of $2 / 3$ is the charged to total pion ratio.

The pseudorapidity density (6) depends on the volume parameter $v_{f}$ and the two temperature scales $T_{f}$ and $T_{d}$ which characterize the collective effect of the system at freeze-out and at chiral transition. The volume parameter $v_{f}$ is centrally colliding energy dependent, it can be fixed by experimental data. From the lattice simulations of QCD which make measurements of the Polyakov loop and the scalar quark density, the deconfinement and chiral transitions coincide at a temperature of about $T_{c}=170 \mathrm{MeV}$ [14]. In the QCD phase diagram [15] the thermal freeze-out temperature in high energy nuclear collisions is about $T_{f}=120 \mathrm{MeV}$. As is well known, the pion mass is approximately temperature independent in the whole chiral breaking phase, we can take $m_{\pi}\left(T_{f}\right)=m_{\pi}(0)=140 \mathrm{MeV}$ at freeze-out and $m_{\sigma}\left(T_{c}\right)=2 m_{\pi}\left(T_{c}\right)=2 m_{\pi}(0)$ at chiral phase transition.
With the given $T_{f}$ and $T_{c}$, the ratio of the pions produced by sigma decay to the direct pions is

$$
r=2\left(\frac{T_{f}}{T_{c}}\right)^{3} \frac{n_{\sigma}\left(T_{c}\right)}{n_{\pi}\left(T_{f}\right)}=52 \%,
$$

this shows a significant influence of the chiral phase transition on the final state pions in relativistic heavy-ion collisions.

In above study of pion multiplicity we did not distinguish the nonthermal sigma decay from thermal sigma decay. As we discussed qualitatively, the thermal and nonthermal pions have very different momentum spectra. The pions produced by sigma decay in the temperature region $T_{f}<T<T_{c}$ have time to thermalize before freeze-out, they lead to an enhancement of thermal pions, while in the interval $T<T_{f}$ the produced pions do not get a chance to thermalize, they have almost the same momentum $p_{0}=\sqrt{\frac{m_{\sigma}^{2}(0,0)}{4}-m_{\pi}^{2}(0,0)}$ in the rest frame of sigma due to the weak temperature dependence of nonstrangle hadron masses at low temperatures. Therefore, if we take a narrow momentum window around $p_{0}$, only a small part of the thermal pions including the direct pions and thermal pions from sigma decay distribute in the window, but all the nonthermal pions are deposited it. The contribution from sigma decay to final state pions is therefore greatly amplified in the window. This strong pion enhancement can be taken as a signature of chiral phase transition in relativistic heavy-ion collisions.

Considering a symmetric window $\Delta p$ around $p_{0}$ and taking the thermal fraction of the pions produced by sigma decay to be $\beta$, the pseudorapidity density in the mid-rapidity region and in the momentum window is

$$
\begin{aligned}
& \frac{\left.\frac{d N}{d \eta}\right|_{\Delta \eta, \Delta p}}{0.5\left\langle N_{\text {part }}\right\rangle}=\frac{1}{2} \frac{2}{3} v_{f} \times \\
& \quad\left[\left.\left(1+2 \beta\left(\frac{T_{f}}{T_{c}}\right)^{3} \frac{n_{\sigma}\left(T_{c}\right)}{n_{\pi}\left(T_{f}\right)}\right) n_{\pi}\left(T_{f}\right)\right|_{\Delta \theta, \Delta p}\right. \\
& \left.\quad+\left.2(1-\beta)\left(\frac{T_{f}}{T_{c}}\right)^{3} \frac{n_{\sigma}\left(T_{c}\right)}{n_{\pi}\left(T_{f}\right)} n_{\pi}\left(T_{f}\right)\right|_{\Delta \theta}\right]
\end{aligned}
$$

with the pion number density

$$
\left.n_{\pi}\left(T_{f}\right)\right|_{\Delta \theta, \Delta p}=\int_{\Delta \theta, \Delta p} \frac{d^{3} \mathbf{p}}{(2 \pi)^{3}} \frac{3}{e^{\epsilon_{\pi} / T_{f}}-1}
$$

in the window.

From (9) the pion enhancement factor due to thermal and nontheral sigma decay in the window is

$$
\begin{aligned}
r_{\Delta p}= & 2 \beta\left(\frac{T_{f}}{T_{c}}\right)^{3} \frac{n_{\sigma}\left(T_{c}\right)}{n_{\pi}\left(T_{f}\right)}+ \\
& 2(1-\beta)\left(\frac{T_{f}}{T_{c}}\right)^{3} \frac{n_{\sigma}\left(T_{c}\right)}{n_{\pi}\left(T_{f}\right)} \frac{\left.n_{\pi}\left(T_{f}\right)\right|_{\Delta \theta}}{\left.n_{\pi}\left(T_{f}\right)\right|_{\Delta \theta, \Delta p}},
\end{aligned}
$$


it depends on the thermal fraction $\beta$ and the momentum window width $\Delta p$.

In our numerical calculation we take the sigma mass in the vacuum $m_{\sigma}(0,0)=600 \mathrm{MeV}$, the corresponding momentum of the nonthermal pions is then $p_{0}=265 \mathrm{MeV}$. For a symmetric momentum window around $p_{0}$ the enhancement factor $r_{\Delta p}$ is shown in Fig.(1) as a function of the window width for three values of the thermal fraction $\beta$ and in Fig.(2) as a function of the thermal fraction $\beta$ for three values of the window width $r_{\Delta p}$. The enhancement drops down rapidly with increasing window width and becomes saturated when the width is larger than about $0.4 \mathrm{GeV}$. It is inversely proportional to the thermal fraction. In the limit of wide window or large thermal fraction, the nonthermal sigma decay can be neglected and the enhancement in the window approaches the total enhancement (8). It is clear to see that the pion number in a small momentum window increases dramatically due to nonthermal sigma decay. Even for $\beta=0.9$, namely only $10 \%$ of the sigmas decay after the freeze-out of the system, the enhancement in a narrow window is still significant.

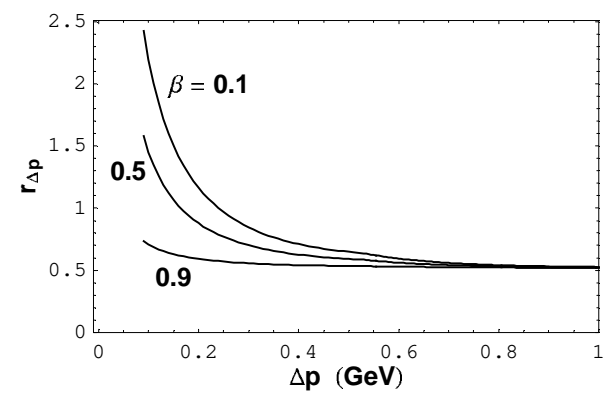

FIG. 1. The window width dependence of the enhancement factor (11) for $\beta=0.1,0.5$ and 0.9 .

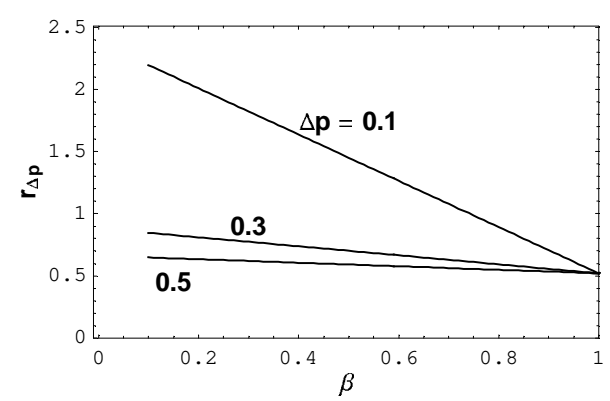

FIG. 2. The thermal fraction dependence of the enhancement factor (11) for $\Delta p=0.1,0.3$ and $0.5 \mathrm{GeV}$.

In summary, we have investigated the effect of chiral phase transition on final state pion spectra. The sigma enhancement at chiral phase transition leads to a significant enhancement of pions due to the sigma decay in chiral symmetry breaking phase. Especially, the sigma decay after the freeze-out of the system results in a cru- cial enhancement in a small momentum window around $p_{0}=\sqrt{\frac{m_{\sigma}^{2}}{4}-m_{\pi}^{2}} \sim 260 \mathrm{MeV}$. This anomalous enhancement comes from the temperature effect which does not affect the sigma mass at low temperatures. For density effect, the sigma mass drops down rapidly even at low densities, the pions from sigma decay after the freeze-out distribute also in a wide momentum region, then the pion enhancement becomes weak in the window. Therefore, in central region of relativistic heavy-ion collisions where there is almost no density effect, the pion enhancement in a small window around the nonthermal momentum $p_{0}$ may be considered as a signature of the chiral phase transition. Although the sigma width and the sigma momentum which are not taken into account in our estimation will smooth the sharp peak around $p_{0}$, we expect that the dynamics of chiral phase transition (here via temperature dependent masses) plays still an essential role in the pion spectra.

acknowledgments: This work was supported in part by the NSFC under contract numbers 19925519 and 10135030, and by the Major State Basic Research Development Program under contract number G2000077407.

[1] For instance, see Proceedings of QM'01, Nucl. Phys. A698(2002).

[2] For instance, see Quark-Gluon Plasma, ed. R. C. Hwa (World Scientific, 1990).

[3] Chungsik Song, Volker Koch, Phys. Lett. B404(1997)1.

[4] M.K.Volkov, E.A.Kuraev, D.Blaschke, G.Röpke, S.Schmidt, Phys. Lett. B424(1998)235.

[5] H.Arthur Weldon, Phys. Lett. B274(1992)133.

[6] S.Chiku and T.Hatsuda, Phys. Rev. D57(1998)R6.

[7] M.Rho, Proceedings of the International Workshop XXIII on Gross properties of Nuclei and Nuclear Excitations, Hirschegg, Austria, January, 16-21, 1995.

[8] P.Zhuang, J.Hüfner, and S.P.Klevansky, Nucl. Phys. A576(1994)525.

[9] R.Rapp, T.Schäfer, E.Shuryak and M.Velkovsky, Phys. Rev. Lett.81(1998)53; M.Stephanov, K.Rajagopal and E.Shuryak, Phys. Rev. Lett. 81(1998)4816; K.Rajagopal, hep-ph/9808348; J.Berges and K.Rajagopal, Nucl. Phys. B538(1999)215.

[10] M.Stephanov, K.Rajagopal and E.Shuryak, Phys. Rev. D60(1999)114028.

[11] P.Zhuang and Z.Yang, Phys. Rev. D63(2001)016004.

[12] C.M.Ko, Z.G.Wu, L.H.Xia and G.E.Brown, Phys. Rev. Lett.66(1991)2577; Phys. Rev. C43(1991)1881; P.Braun-Munzinger, J.Stachel, Nucl. Phys. A606(1996)320.

[13] J.D.Bjorken, Phys. Rev. D27(1983)140.

[14] F.Karsch, Nucl. Phys. B(Proc. Suppl.)83-84(2000)14.

[15] U.Heinz, Nucl. Phys. A685 (2001) 414. 